\title{
Computed tomography and magnetic resonance imaging assessment of aortic valve stenosis: an update
}

\author{
Yeon Hyeon Choe ${ }^{1,2}$, Sung Mok Kim ${ }^{1,2}$, Sung-Ji Park ${ }^{2,3}$
}

\author{
${ }^{1}$ Department of Radiology, Samsung Medical Center, Sungkyunkwan University School of Medicine, Seoul, Korea \\ ${ }^{2}$ HVSI Imaging Center, Heart Vascular Stroke Institute, Samsung Medical Center, Sungkyunkwan University School of Medicine, \\ Seoul, Korea \\ ${ }^{3}$ Division of Cardiology, Department of Medicine, Samsung Medical Center, Sungkyunkwan University School of Medicine, Seoul, \\ Korea
}

Received: June 7, 2020

Revised: July 12, 2020

Accepted: July 16, 2020

Corresponding author:

Yeon Hyeon Choe

Department of Radiology,

Samsung Medical Center,

Sungkyunkwan University

School of Medicine, 81 Irwon-ro,

Gangnam-gu, Seoul 06351, Korea

Tel: +82-2-3410-2509

E-mail:yhchoe@skku.edu

\begin{abstract}
Computed tomography (CT) and magnetic resonance imaging (MRI) are being increasingly utilized for the evaluation of cardiac valves. Although echocardiography is the first-line imaging modality in the evaluation of aortic stenosis (AS), CT and MRI can be adopted as adjunct imaging modalities for assessing the severity of AS. First, CT has established its role in the evaluation of co-existing coronary artery disease in AS patients, while stress MRI can be employed to exclude myocardial perfusion abnormalities. For the pre- and post-procedural evaluation of transcatheter aortic valve implantation or replacement, $\mathrm{CT}$ plays a very important role in determining the size of prosthetic valves and evaluating post-procedural complications. $\mathrm{CT}$ also helps assess the prosthetic valve sizes with 3-dimensional printing. Late gadolinium enhancement, T1 mapping, and feature tracking of left ventricular myocardium enable assessment of left ventricular function and myocardial fibrosis in patients with AS. Four-dimensional flow analysis gives new insights on flow patterns, kinetic energy, and wall shear stress in the ascending aorta in AS patients. In summary, CT and MRI are playing increasingly important roles in the evaluation of aortic valve disease.
\end{abstract}

Keywords: Aortic valve stenosis; Magnetic resonance imaging; Tomography, X-ray computed; Transcatheter aortic valve replacement
This is an Open Access article distributed under the terms of the Creative Commons Attribution Non-Commercial License (https:// creativecommons.org/licenses/ by-nc/4.0/).

\section{INTRODUCTION}

Recently, computed tomography (CT) and magnetic resonance imaging (MRI) are being utilized for the assessment of aortic valve disease (Figs. 1-7) [1-8]. Recent developments in CT enable simultaneous evaluation of the coronary arteries, aorta, proximal lower extremity arteries 

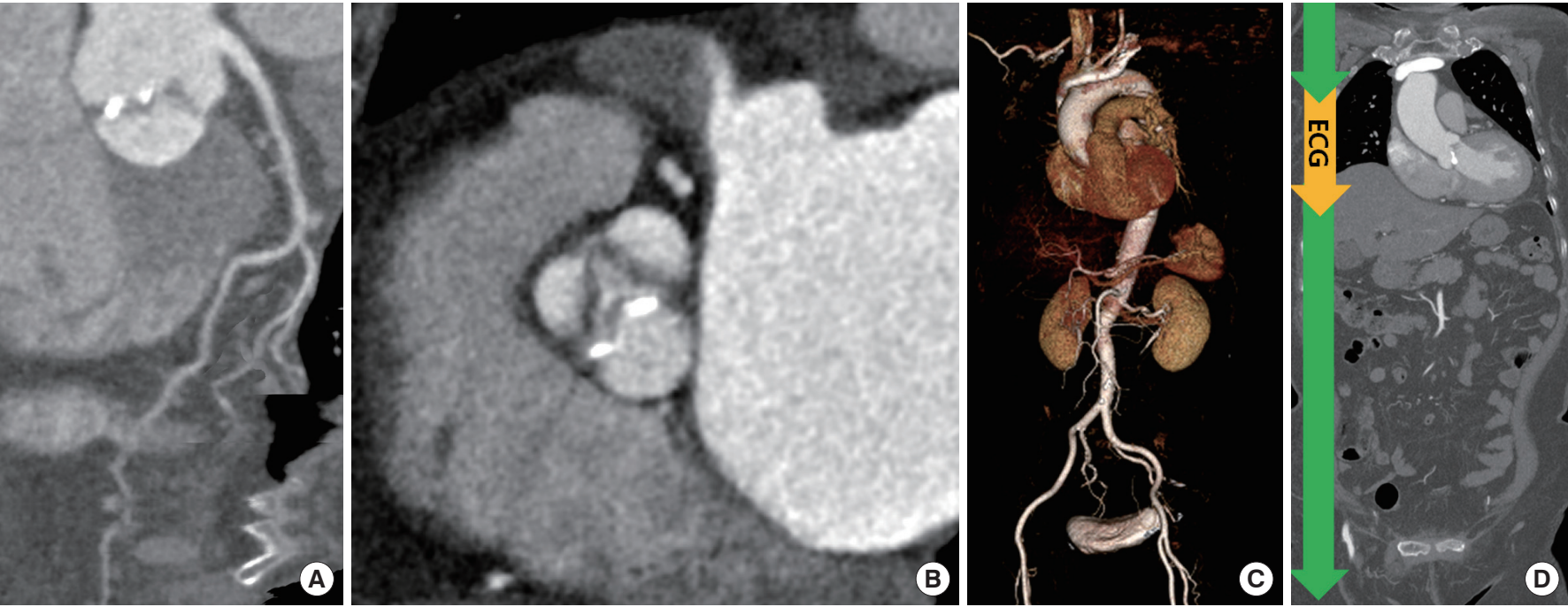

Fig. 1. Variable helical pitch computed tomography (CT) technique in a patient with aortic stenosis. Simultaneous preoperative evaluation of coronary artery (A), aortic valve (B), aorta, arch vessels, and iliofemoral arteries (C) is performed using this technique with single injection of contrast material within one scan. (D) Electrocardiography (ECG)-gated CT angiography is performed in the cardiac area with a heart-dedicated pitch (0.15) and non-ECG-gated scan is performed in the upper chest and abdominopelvic area with a higher pitch (0.81).
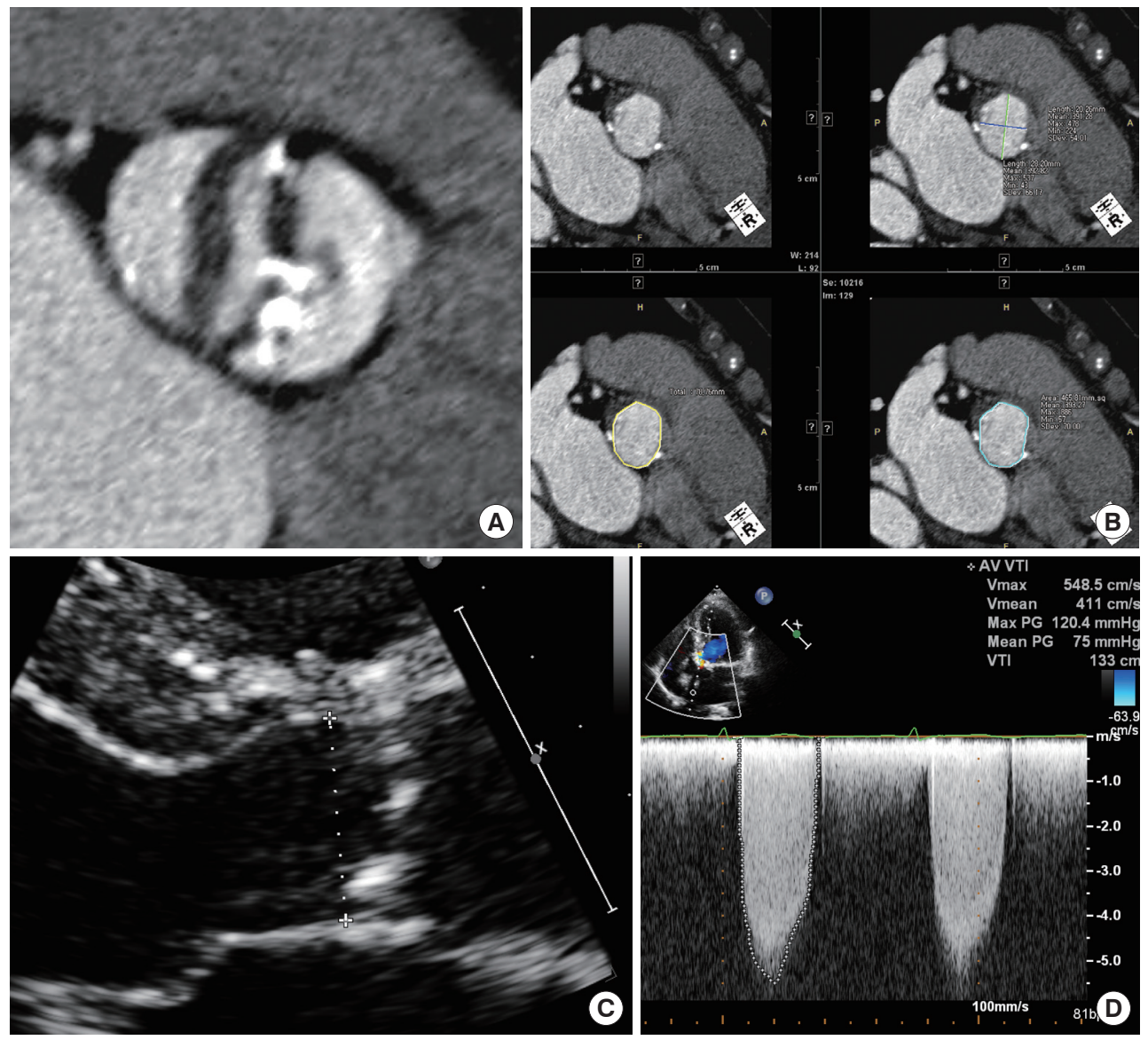

Fig. 2. Comparison of computed tomography (CT) and transthoracic echocardiography in the evaluation of aortic stenosis in a patient with bicuspid aortic valve. Mid-systolic-phase CT shows aortic valve area (AVA) of $0.87 \mathrm{~cm}^{2}$ (A) and left ventricular outflow tract (LVOT) (B) dimensions of $4.65 \mathrm{~cm}^{2}$ (area) and $20.3 \times 28.2 \mathrm{~mm}$ (diameter). At transthoracic echocardiography, AVA was calculated as $0.52 \mathrm{~cm}^{2}$ and LVOT, $23.9 \mathrm{~mm}$ (C) (area, $4.49 \mathrm{~cm}^{2}$ ) with peak systolic velocity of $5.5 \mathrm{~m} / \mathrm{sec}$ and mean pressure gradient of $75 \mathrm{~mm} \mathrm{Hg}$ (D). AVA corrected by CT-derived LVOT area was $0.54 \mathrm{~cm}^{2}$. 

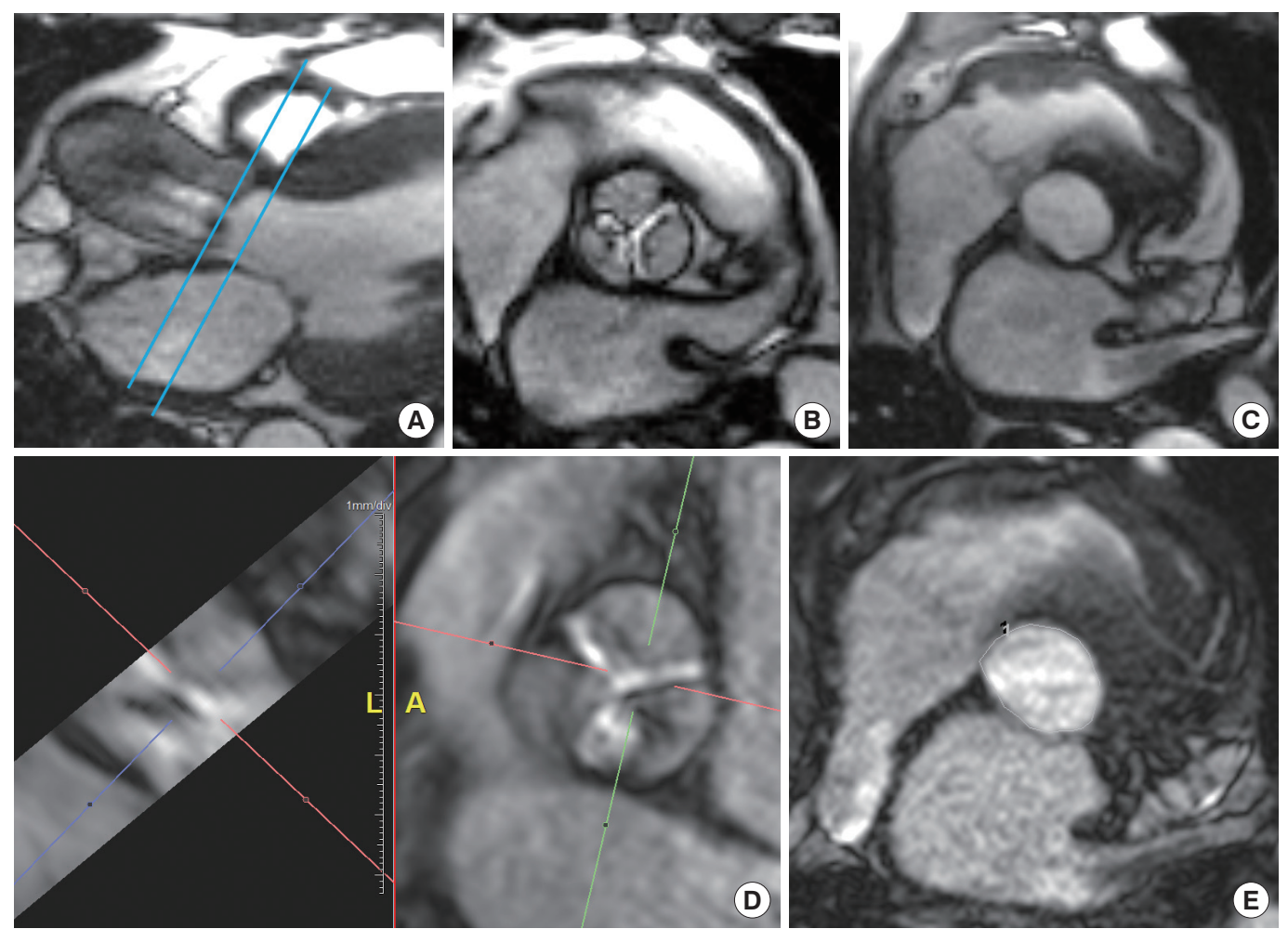

Fig. 3. Magnetic resonance imaging (MRI) measurement of planimetric aortic valve area (AVA) and left ventricular outflow tract (LVOT) area in a 65-year-old male with moderate aortic stenosis on echocardiography. Cine MRI slices of 6-mm slice thickness should be prescribed precisely at the levels of tips of aortic valve cups and LVOT on the mid-systolic 3-chamber view cine MRI (A) for two-dimensional (2D) planimetry of AVA (B) and LVOT (C). In the three-dimensional (3D) approach, high-resolution 3D data (volume of 10 2-mm-slices, 20-mm thickness of the whole volume; matrix, $256 \times 209$; 3D balanced steady-state free precession at 1.5T) are obtained during mid-systolic phase in one breath hold. AVA measured was $0.94 \mathrm{~cm}^{2}$ with $3 \mathrm{D}$ method (D) and $1.02 \mathrm{~cm}^{2}$ with $2 \mathrm{D}$ method, while it was $1.21 \mathrm{~cm}^{2}$ with echocardiography using continuity equation. LVOT was $5.25 \mathrm{~cm}^{2}(22.0 \times 28.4 \mathrm{~mm})$ on 3D MRI (E), $5.10 \mathrm{~cm}^{2}(21.7 \times 29.3 \mathrm{~mm})$ on 2D MRI, and $4.48 \mathrm{~cm}^{2}(23.9 \mathrm{~mm})$ on echocardiography. Peak systolic velocity was $3.4 \mathrm{~m} / \mathrm{sec}$ on velocity-encoded cine MRI and $3.5 \mathrm{~m} / \mathrm{sec}$ on echocardiography. AVAs on echocardiography corrected by LVOT at 2D MRI and 3D MRI are 1.38 and $1.41 \mathrm{~cm}^{2}$, respectively. Left ventricular ejection fraction measured $50 \%$ at cine MRI.

with the use of smaller contrast amount and lower radiation dose than before [9-17]. A wide-coverage (256- or 320-slice) detector design or high pitch dual-source CT enables a fast scan covering the aorta, coronary, and proximal femoral arteries (Fig. 1) [18]. Improved detector function and modelbased iterative reconstruction are helpful in reducing the radiation dose of CT. Cardiac magnetic resonance (CMR) can be used to assess the severity of aortic stenosis (AS) and determine the prosthetic valve sizes as a reliable alternative to $C T$ and transesophageal echocardiography (TEE) [6,19-21].

\section{CORONARY ARTERY EVALUATION IN AS PATIENTS}

Coronary computed tomography angiography (CCTA) may play a clinical role as an alternative to invasive coronary angiography (ICA) before cardiac valve surgery or transcatheter aortic valve implantation or replacement (TAVI/TAVR). With the high prevalence of coronary artery disease in patients with severe AS, percutaneous coronary intervention will become increasingly necessary in patients undergoing TAVI [22]. According to a meta-analysis of 13 studies, in 1,498 patients (mean age, 74 years; 47\% men; 76\% TAVI procedures) undergoing surgical aortic valve surgery or TAVI, the pooled prevalence of significant stenosis (>50\%) determined by ICA was 43\% [23]. CCTA showed the pooled sensitivity, specificity, and positive-likelihood and negative-likelihood ratios in detecting significant coronary stenosis, with ICA as the gold standard, of $95 \%, 79 \%, 4.48$, and 0.06 , respectively [23]. In a study involving $140 \mathrm{AS}$ patients (68 males; $82.3 \pm 7.7$ years) undergoing TAVI, the diagnostic performance of CCTA in the low calcium score group (42 [30\%] patients, $<400$ Agatston calcium score) was better than that in the high calcium score group (98/140 [70\%] patients, $\geq 400$ calcium score) (area under re- 

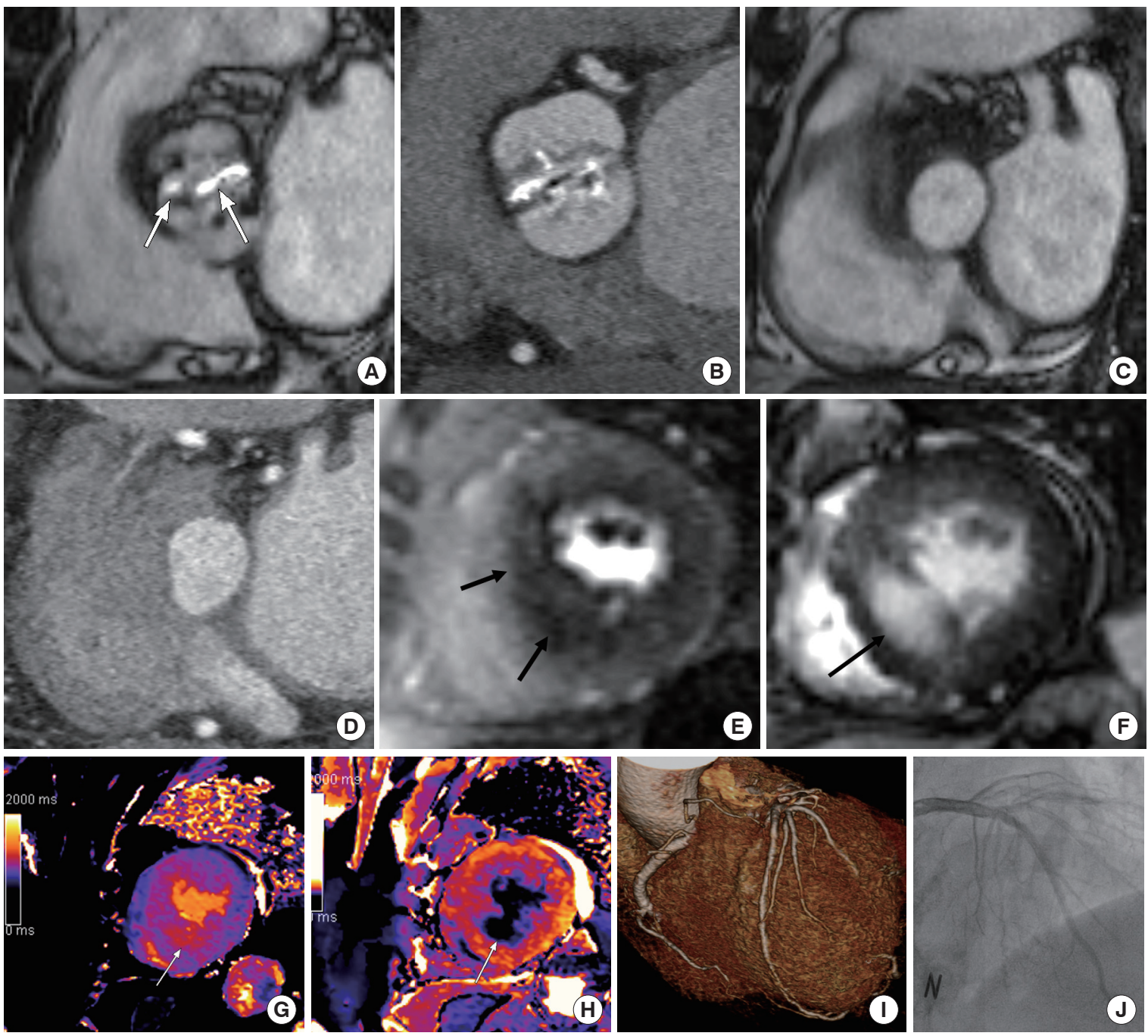

Fig. 4. Comprehensive computed tomography (CT) and cardiac magnetic resonance (CMR) evaluation of aortic valve (AV) and myocardium in a 75-year-old female patient with severe aortic valve stenosis (AS) and bicuspid AV. Mid-systolic cine magnetic resonance imaging shows severe AS with two holes (arrows) with aortic valve area (AVA) of $0.54 \mathrm{~cm}^{2}$ on planimetry (A). AVA was $0.43 \mathrm{~cm}^{2}$ on $C T$ (B) and $0.54 \mathrm{~cm}^{2}$ on echocardiography. Vmax at echocardiography was 6.0 and $4.9 \mathrm{~m} / \mathrm{sec}$ at CMR. Left ventricular outflow tract (LVOT) was $4.35 \mathrm{~cm}^{2}(22.2 \times 26.5 \mathrm{~mm})$ on 2D CMR (C), $3.86 \mathrm{~cm}^{2}(19.7 \times 25.7 \mathrm{~mm})$ on CT (D), and $3.39 \mathrm{~cm}^{2}$ (diameter, $2.08 \mathrm{~cm}$ ) on echocardiography. Left ventricular (LV) ejection fraction was $62 \%$ on CMR. AVAs at echocardiography corrected by LVOT at CMR and at CT were 0.69 and $0.61 \mathrm{~cm}^{2}$, respectively. (E) Adenosine-stress CMR shows patchy hypoperfusion (arrows) in middle inferoseptal wall of LV and milder diffuse hypoperfusion in other segments. Late gadolinium enhancement (LGE) imaging (F), native (G) and postcontrast (15-minute delay, H) T1 mapping using MOLLI technique show abnormal extracelluar volume fraction (0.47) in the area of patchy LGE (arrows). CT angiography (I) and catheter coronary angiography (J) show no significant coronary abnormalities.

ceiver operating characteristic curve [AUC] 0.81 vs. 0.63) [24].

AS patients may have microvascular angina. Semi-quantitative myocardial perfusion reserve index (MPRI) using adenosine-stress CMR could detect impaired coronary microvascular function in patients with AS. According to a study of 117 patients with severe AS, MPRI values were much lower in the angina group than the asymptomatic group $(0.74 \pm 0.25$ vs. $1.08 \pm 0.28, P<0.001)$ and significantly lower in severe AS patients than in normal controls $(0.90 \pm 0.31$ vs. $1.25 \pm 0.21$,
$P<0.001)$ [25]. As per logistic regression analysis, the only independent predictor for angina was MPRI (odds ratio, 0.003; $P<0.001)$. On multivariate analysis, left ventricular mass index (LVMI) was found to be the strongest contributing factor to MPRI (standardization coefficient, $-0.428 ; \mathrm{P}<0.001$ ) [25].

\section{PRE-AND POST-TAVI EVALUATION WITH CT}

Recent $\mathrm{CT}$ techniques enable comprehensive evaluation before and after TAVI/TAVR in one breath hold with a variable or 

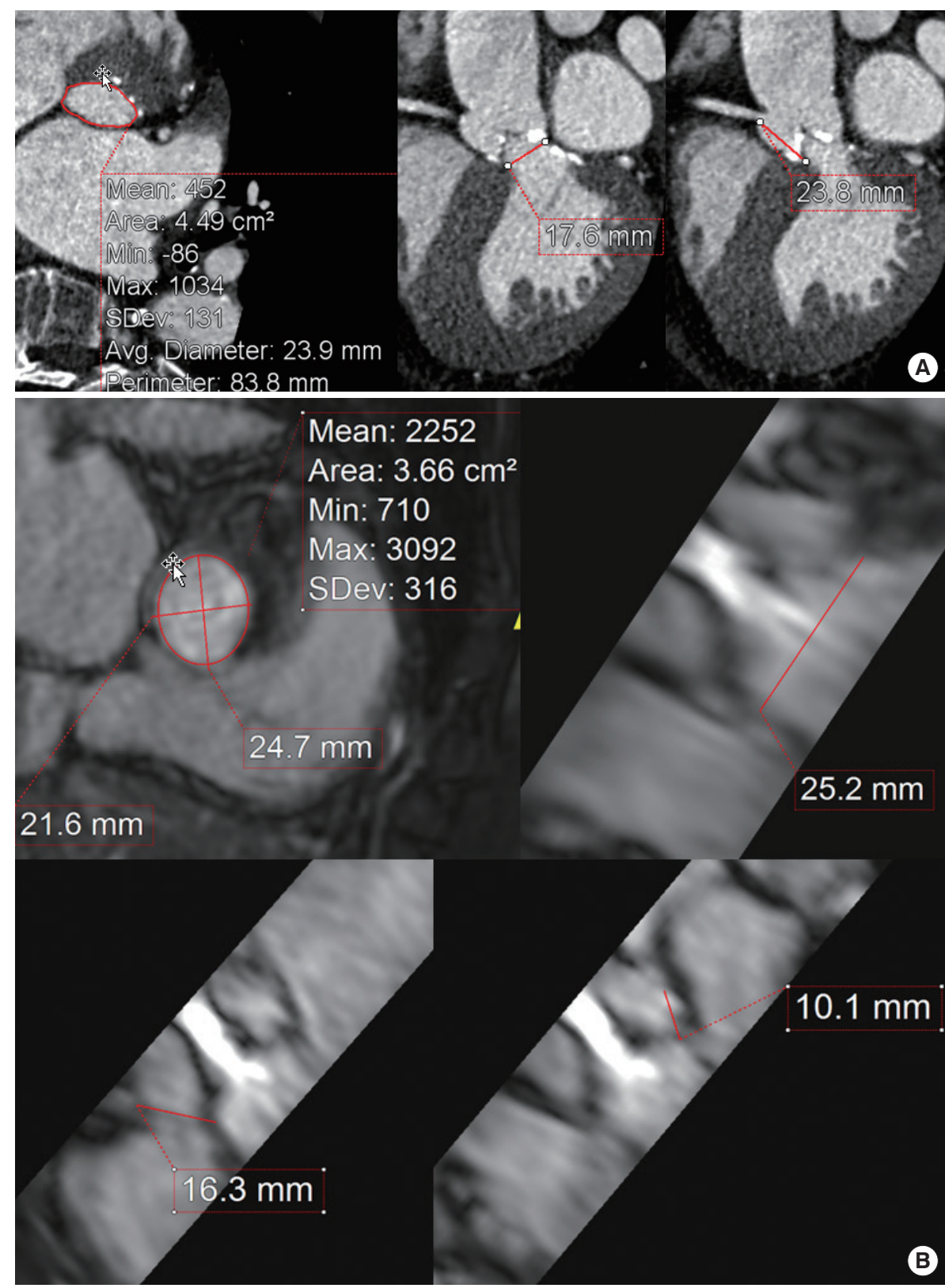

Fig. 5. Computed tomography (CT) and magnetic resonance imaging measurements for pre-transcatheter aortic valve implantation evaluation in different patients. CT is commonly used for the preoperative evaluation of aortic valve (AV) annulus and distance from the AV annulus to coronary ostia (A). Similarly, three-dimensional volume data from cardiac magnetic resonance can be used for those measurements (B).

high helical pitch [7,9,13,14,16]. The CT area showed the highest correlation (0.932) among CT and TEE estimates, as compared with surgical annulus measurements [26].

The use of CCTA in addition to echocardiographic data in selecting the transcatheter heart valve (THV) size reduced the long-term occurrence of cardiovascular events [27]. Imaging strategy with CCTA was found to optimize and improve the outcome of TAVI. According to Casset et al. [27], multidetector-CT (MDCT) integration group improved the TAVI outcome compared with echocardiography only group ( $8 \%$ vs. $40 \%$, $P=0.008$ ), mainly by decreasing the rate of paravalvular aortic regurgitation (PAR, $28 \%$ vs. $4 \%, P=0.04$; major vascular complications $12 \%$ vs. $4 \%, P=0.6$; all-cause death $16 \%$ vs. $4 \%, P=0.34$; no stroke).

CCTA data and echocardiographic outcome data of the PARTNER II (Placement of AoRTic TraNscathetER Valves Trial II) SAPIEN 3 (Edwards Lifesciences, Irvine, CA, USA) intermediate-risk cohort of 835 patients showed that for the SAPIEN 

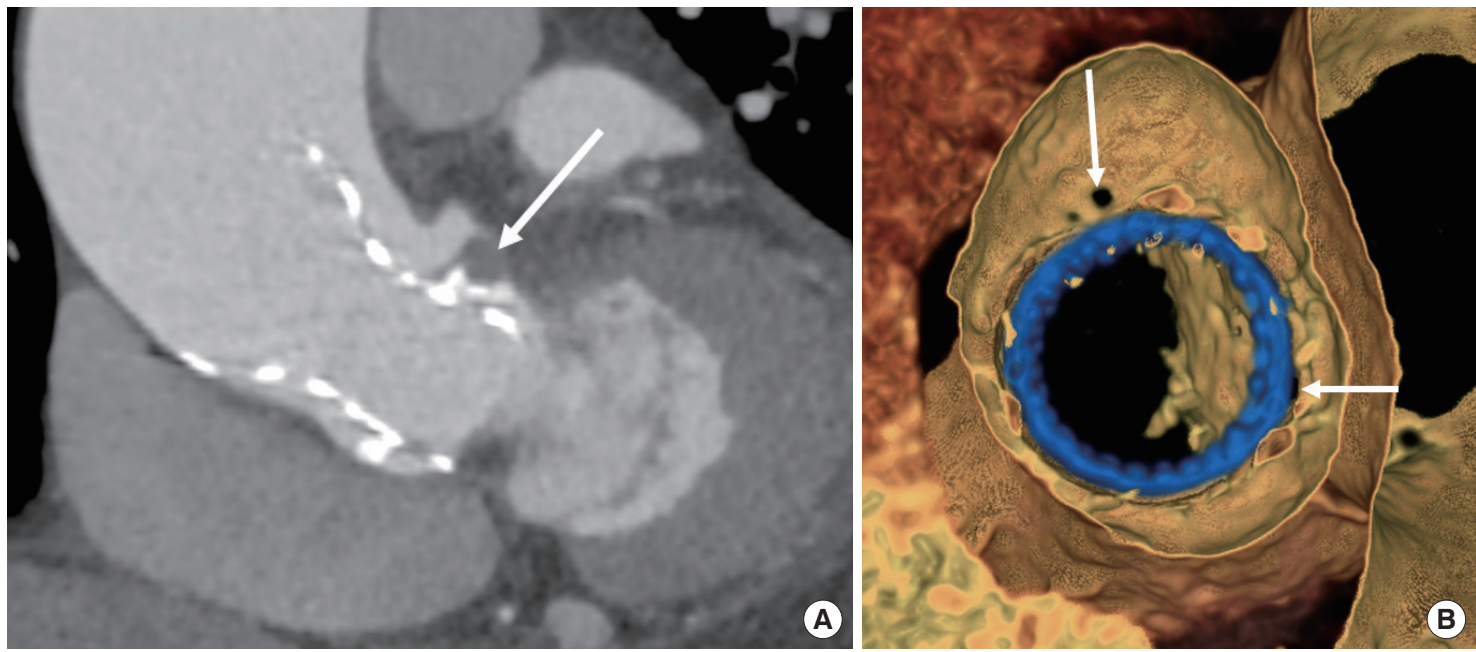

Fig. 6. Computed tomography evaluation of transcatheter aortic valve implantation (TAVI). (A) Thrombus (arrow) 4 months after TAVI. (B) Paravalular aortic regurgitation (arrows).
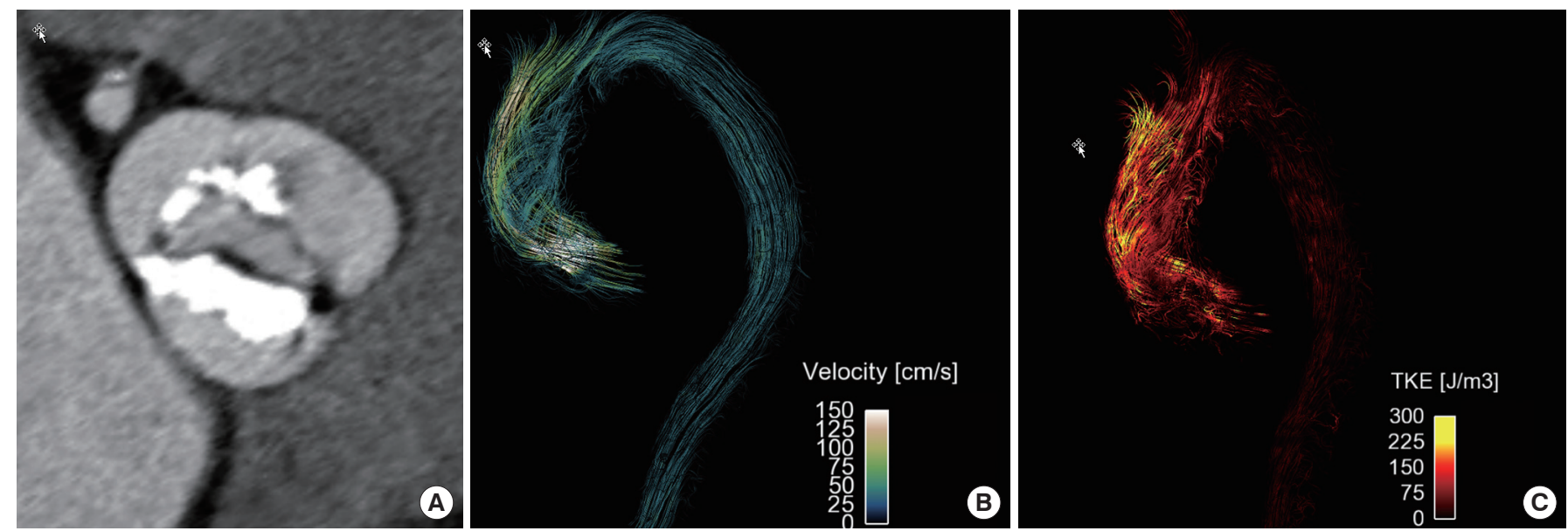

Fig. 7. Four-dimensional magnetic resonance flow assessment of the ascending aorta in a 73-year-old male patient with aortic valve stenosis due to bicuspid aortic valve (AV). Computed tomography shows bicuspid AV with calcifications (A). Echocardiography showed mean pressure gradient of $79 \mathrm{~mm} \mathrm{Hg}$ (peak, $132 \mathrm{~mm} \mathrm{Hg}$ ) and peak systolic velocity of $5.8 \mathrm{~m} / \mathrm{sec}$. Flow mapping indicates the streamline of the flow in the ascending aorta (B). Increased velocity and helical flow pattern are noted in the ascending aorta. The turbulent kinetic energy is visualized by the volume rendering within the entire thoracic aorta (C). The turbulent kinetic energy (TKE) is increased in the ascending aorta and aortic arch. Images courtesy of Dong Hyun Yang, MD, Asan Medical Center.

3 THV, the frequency and extent of PAR was inversely related to the degree of oversizing, and acceptable rates of PAR were achieved at low degrees of oversizing [28]. Perimeter and area oversizing conferred similar predictive capacity in regard to the occurrence of PAR after THV implantation (AUC, $0.78[95 \% \mathrm{Cl}, 0.70$ to 0.85$]$ vs. AUC, $0.78[95 \% \mathrm{Cl}, 0.72$ to 0.85$]$; $\mathrm{P}<0.001$ ) [28]. In patients with area oversizing of $\geq 10 \%$, moderate or high PAR was observed infrequently (0.3\%). For low degrees of area oversizing, the rate of moderate or high PAR increased to $4.2 \%$ (for oversizing of $\geq 5 \%$ to $10 \%$ ) and
$2.8 \%$ (for oversizing of $\geq 0 \%$ to $5 \%$ ). The rate of moderate or high PAR increased to $7.8 \%$ for area undersizing of $-5 \%$ to $0 \%$, and further increased to $13.2 \%$ for undersizing below $-5.0 \%$. Similarly, a PAR rate of $2.4 \%$ was found for $0 \%$ to $2.5 \%$ perimeter oversizing and of $0.6 \%$ for $\geq 2.5 \%$ perimeter oversizing. With perimeter undersizing down to $-2.5 \%$, the rate of moderate or high PAR increased to $6.8 \%$ and then further increased to $9.4 \%$ for pronounced undersizing of below $-2.5 \%$. The optimal cutoff for predicting moderate or high PAR was $0.05 \%$ for area oversizing and $-1.24 \%$ for perimeter oversizing. 
Moderate or severe landing zone calcifications had a relative risk of $1.36(95 \% \mathrm{Cl}, 1.09$ to $1.69 ; \mathrm{P}=0.006)$ for mild or higher PAR compared to none or mild landing zone calcifications.

Three-dimensional (3D)-printing based on CT data is a unique patient-specific method, allowing a tailored and individualized approach for aortic valve-sparing root reconstruction surgery [29]. With optimization, 3D models may help predict PAR in specific patients [30]. According to Ripley et al. [30], aortic root 3D models were highly accurate, with excellent agreement between annulus measurements made by 3D models and those made by corresponding 2D data (mean difference, $-0.34 \mathrm{~mm}$; $95 \%$ limits of agreement, $\pm 1.3 \mathrm{~mm}$ ). Examination of the fit of valves within aortic root 3D models correctly predicted PAR in six of nine patients (six true positives, three false negatives) and absence of PAR in five of seven patients (five true negatives, two false positives).

\section{MRI FOR TAVI EVALUATION}

CMR is useful for TAVI evaluation before the procedure [3133]. CMR can provide incremental information, including advanced tissue characterization with late gadolinium enhancement (LGE) and T1 mapping in patients with AS. In addition, left ventricle (LV) strain analysis with feature tracking and assessment of myocardial perfusion can be performed. CMR is now ready for implementation in clinical practice [20].

In the study of Cannao et al. [34], a non-contrast MRA protocol combining self-navigated 3D (SN3D) radial whole-heart and quiescent-interval single-shot (QISS) pulse sequences for the assessment of cardiac and vascular access route anatomy was technically feasible for TAVI planning. Acquisition time of the combined SN3D and QISS protocol was $10.1 \pm 1.6$ minute. All MRA measurements showed good agreement with $\mathrm{CT}$ angiography (CTA) in patients and there was no difference in qualitative ratings between MRA and CTA. Interobserver agreement was good for MRA (kappa $=0.71$ to 0.76 ) and excellent for CTA (kappa $=0.82$ to 0.84 ).

CMR is considered a reproducible, accurate, and reliable method to assess PAR severity as it allows direct quantification of AR. In a meta-analysis of seven studies by Papanastasiou et al. [35] on the level of agreement between two-dimensional transthoracic echocardiography (2D TTE) and CMR on grading the severity of aortic regurgitation (AR) after TAVI, six studies reported a low correlation between 2D TTE and CMR (kappa coefficient ranging from -0.02 to 0.41 ), whereas one study showed good agreement with a kappa coefficient of 0.72 . The area under the curve for detection of moderate or severe AR with TTE was 0.83 (95\% confidence interval [CI], 0.79 to 0.86 ), with CMR as the gold standard [35]. In the study by Salaun et al. [33], PAR severity was assessed 5 days after TAVI using TTE and CMR in 30 patients (COREVALVE, $n=10$; EDWARDS SAPIEN XT, $n=20$ ). In CMR, regurgitation volume and regurgitation fraction (RF) were significantly correlated with AR severity at TTE $(P<0.001)$, with mean RF values of $9.2 \% \pm 7.6 \%$ in mild $(n=22), 20.3 \% \pm 4.2 \%$ in moderate $(n=3)$, and $46.8 \% \pm 10.8 \%$ in severe PAR patients $(n=5)$. A cutoff value of RF $<14 \%$ on CMR accurately differentiated mild from moderate/severe PAR (sensitivity $100 \%$, specificity 82\%) [33].

\section{AS EVALUATION WITH ECHOCARDIOG- RAPHY, MRI, OR CT}

\section{Limitations of echocardiography}

As per current guidelines, severe AS is defined as the aortic valve area normalized to body surface area (AVA/BSA) $<0.6$ $\mathrm{cm}^{2} / \mathrm{m}^{2}$. According to Tribouilloy et al. [36], AVA/height showed better predictive performance than AVA/BSA with better reclassification and discrimination (net reclassification improvement, 0.33 vs. 0.28 ; integrated discrimination improvement, 0.10 vs. 0.08 ; C statistic, 0.67 vs. 0.65 ) than AVA/weight and AVA/body mass index. Inconsistency of hemodynamic characterization in severe AS between echocardiography (AVA $<1.0 \mathrm{~cm}^{2}$ ) and catheterization (mean pressure gradient $>40 \mathrm{~mm} \mathrm{Hg}$ ) is observed in about half of the patients, and this is only partly explained by low-flow (low LV ejection fraction [EF]) conditions [37]. This discordance can be also explained by underestimation of left ventricular outflow tract (LVOT) diameter or velocity-time integral. Doppler assessment may fail to accurately measure AVA in low-gradient AS patients and the coexistence of low-flow and a para doxically preserved LVEF (PLF-LG AS), is a real and frequently occurring entity (5\% to 20\%) [38]. Projected AVA at normal flow rate $(250 \mathrm{~mL} / \mathrm{sec})$ calculated with stress echocardiography using low-dose dobutamine is superior to traditional severity criteria (AVA $<1.0 \mathrm{~cm}^{2}$ and mean gradient $\geq 40 \mathrm{~mm}$ $\mathrm{Hg}$ ) in unmasking severe AS and predicting outcomes in patients with reduced LVEF. However, $\mathrm{AVA}_{\text {proj }}$ is not reliable if the increase in transvalvular flow rate is below $15 \%$ and dobutamine-stress echocardiography may not be able to induce a significant increase in flow rate [38].

CT measurement of aortic valve area and calcium According to the meta-analysis of nine studies (175 women 
and 262 men), MDCT is an efficient method for obtaining accurate AVA measurements in patients with AS [39]. The mean AVA as measured by CT was $1.0 \pm 0.1 \mathrm{~cm}^{2}$ and the mean AVA measured by TTE was $0.9 \pm 0.1 \mathrm{~cm}^{2}$ [39]. The correlation between CT and TTE AVA measurements was strong $(r=0.89)$ and the mean difference was $0.03 \pm 0.05 \mathrm{~cm}^{2}$. Because of the elliptical anatomy of LVOT, 2D TTE may underestimate the LVOT area. Fusion of the LVOT area measured by CT and echocardiographic flow data has been proposed to improve the assessment of AS severity. For hemodynamic reasons, anatomic AVA is larger than effective AVA. Clavel et al. [40] calculated AVA corrected by LVOT area measured with CT using the following equation: $\mathrm{AVA}_{\mathrm{CT}}=(\mathrm{LVOT}$ area on $\mathrm{CT}) \times \mathrm{VTI}_{\mathrm{LVOT}} /$ $\mathrm{VTI}_{\mathrm{AO}}$, where $\mathrm{VTI}_{\text {LVOT }}$ is the velocity-time integral of LVOT and $\mathrm{VTI}_{\mathrm{AO}}$ is the VTI of stenotic aortic valve. In a study of $269 \mathrm{pa}-$ tients ( $76 \pm 11$ years of age, $61 \%$ men) with isolated calcific AS (mean gradient $44 \pm 18 \mathrm{~mm} \mathrm{Hg}$; EF $58 \% \pm 15 \%$ ) who had undergone Doppler echocardiography and MDCT within the same episode of care, the head-to-head comparison of AVA with $C T\left(A V A_{C T}\right)$ and with Doppler echocardiography $\left(\mathrm{AVA}_{\text {Echo }}\right)$ revealed that $A V A_{C T}$ was larger than $A V A_{\text {Echo }}$ (difference, 0.12 $\pm 0.16 \mathrm{~cm}^{2} ; \mathrm{P}<0.001$ ) but did not improve outcome prediction [40]. For long-term survival, after multivariable adjustment, $A V A_{E c h o}$ and $A V A_{C T}$ were independently predictive (hazard ratio [HR], 1.26; $95 \% \mathrm{Cl}, 1.13$ to $1.42 ; \mathrm{P}<0.001$ vs. HR, 1.18; $95 \% \mathrm{Cl}, 1.09$ to 1.29 per $0.10 \mathrm{~cm}^{2}$ decrease, respectively; $P<0.001)$ with a similar prognostic value $(P \geq 0.80)[40]$. Large cut-point values should be used for severe AS, if $A V A_{C T}$ $\left(<1.2 \mathrm{~cm}^{2}\right)$ is compared with $\mathrm{AVA}_{\text {Echo }}\left(<1.0 \mathrm{~cm}^{2}\right)$ [40].

Jander et al. [41] compared effective AVA (calculated from the continuity equation using CT-LVOT and TTE Doppler measurements) with anatomic AVA based on CT planimetry findings of 244 consecutive patients (mean age, $81 \pm 5$ years; $61 \%$ female) with AS. Substituting the LVOT area from TTE by the CT-LVOT resulted in an increase in AVA from $0.74 \pm 0.15$ to $0.92 \pm 0.18 \mathrm{~cm}^{2}(P<0.01)$, which was larger than anatomic AVA from CT $\left(0.82 \pm 0.15 \mathrm{~cm}^{2}\right)$ and AVA from TEE planimetry $\left(0.79 \pm 0.14 \mathrm{~cm}^{2}, P<0.01\right.$ vs. CT-LVOT). In the subgroup $(n=67)$ presenting with low-gradient severe $A S$ and preserved $E F$, the results were similar (AVA from TTE, $0.76 \pm 0.09$; from CT-LVOT, $0.97 \pm 0.14$; CT planimetry, $0.86 \pm 0.12$; TEE planimetry, $0.82 \pm 0.13 \mathrm{~cm}^{2}$ ). Aortic valve calcification (AVC) score quantitated by $\mathrm{CT}$ is helpful for identifying true severe AS by applying thresholds of 2,000 and 1,200 arbitrary units, respectively, for men and women. This modality should be considered, particularly if stress echocardiography is either not feasible or inconclusive [38]. Sex-specific CT-AVC thresh- olds enable identification of severe AS and provide prognostic information. In a multicenter study of 918 patients, CTAVC provided excellent discrimination for severe AS (C statistic: women 0.92, men 0.89). The optimal sex-specific CT-AVC thresholds (Agatston score: women 1,377, men 2,062) were nearly identical to those previously reported (Agatston score: women 1,274, men 2,065) [42]. Sex-specific CT-AVC thresholds independently predicted aortic valve replacement (AVR) and death (HR, 3.90; 95\% Cl, 2.19 to 6.78; $\mathrm{P}<0.001)$ after adjustment for age, sex, peak velocity, and AVA in 215 patients with available clinical outcomes [42].

\section{CMR assessment of AS severity}

According to a systemic review, eight of 12 papers found CMR for the diagnosis of AS to have excellent reliability and reproducibility (low inter- and intraobserver variability) [2]. One study noted a sensitivity and specificity of $96 \%$ and $100 \%$, respectively, when using CMR to detect AS diagnosed by cardiac catheterization (CC). Another study noted a lower sensitivity and specificity of $78 \%$ and $89 \%$, respectively, which were better than the values reported when using TEE or TTE to detect severe AS, as noted on CC.

A head-to-head comparison between cine CMR and echocardiography for grading of AS severity revealed similar results between them, where AVA was the largest with planimetric CMR (0.93 $\pm 0.42 \mathrm{~cm}^{2}$ ) (TEE, $0.92 \pm 0.32$; continuity equation by CMR, $0.86 \pm 0.30$; TTE, $0.78 \pm 0.25 \mathrm{~cm}^{2}$ ) [43]. CMR planimetry strongly correlated with TEE planimetry (concordance correlation coefficient [CCC], 0.85; 95\% Cl, 0.75 to 0.91 ). The aortic valve effective orifice area obtained by the continuity equation CMR was similar to that obtained by TTE (CCC, $0.82 ; 95 \% \mathrm{Cl}, 0.68$ to 0.90 ).

According to Da Silveira et al. [44], multi-directional flow MRI, which encodes all three components of the velocity vector, can potentially outperform TTE in AS patients with eccentric or multiple jets. They compared fast 3-direction (3Dir) and 1-direction (1Dir) phase-contrast (PC) and TTE. The correlations ranged from 0.61 to 0.81 between TTE and 1Dir-PCCMR parameters and from 0.61 to 0.87 between TTE and 3Dir-PC CMR parameters. The correlation coefficients between TTE and 1Dir and 3Dir-PC CMR V $V_{\text {peak }}$ were 0.81 and 0.87 , respectively. Despite that, the comparison of Pearson's correlations of the techniques did not reach statistical significance, even in the sub-analysis of severe cases $(P>0.05)$ [44].

Significant overestimation of transvalvular peak pressure drops occur with Doppler echocardiography because of approximation of blood flow as a single streamline. Donati et al. 
[45] assessed the accuracy of the Bernoulli principle in estimating the peak pressure drop at aortic valve using 3D CMR flow data in 32 subjects. Analysis of the pressure components confirmed that the spatial acceleration of the blood jet through the valve is the most significant aspect (accounting for $99 \%$ of the total drop in stenotic subjects). However, the Bernoulli formulation showed a consistent overestimation of the transvalvular pressure (average of 54\%; range, $5 \%$ to $136 \%$ ) resulting from the use of a single peak velocity value, which neglects the velocity distribution across the aortic valve plane. This assumption was the source of uncontrolled variability. A corrected formulation that accounts for the cross-sectional profile of the blood flow was proposed by the authors and adapted to both CMR and echocardiographic data [45].

\section{MYOCARDIAL FIBROSIS}

Myocardial fibrosis (MF) is regarded as an early and objective marker of left ventricular decompensation, particularly in asymptomatic AS patients [46]. Replacement MF or diffuse MF can be evaluated by LGE and T1 mapping [5,47-50]. Treibel et al. [51] investigated MF in 133 patients with severe, symptomatic AS using invasive biopsy and non-invasive imaging. Three patterns of MF were identified: (1) thickened endocardium with a fibrotic layer, (2) microscopic scars, with a subendomyocardial predominance, and (3) diffuse interstitial fibrosis. Collagen volume fraction (CVF) was elevated $(P<0.001)$, as compared with controls, and higher $(P<0.001)$ in endocardium-containing samples, with a decreasing CVF gradient from the subendocardium $(P=0.001)$. LGE in grams, by the 3-standard deviation method, correlated with CVF $(P<0.001)$ but not ECV. Both LGE and extracellular volume (ECV) correlated independently $(\mathrm{P}<0.001)$ with $\mathrm{N}$-terminal pro-brain natriuretic peptide and high-sensitivity troponin $\mathrm{T}$ levels. Combined high ECV and LGE better identified patients with adverse LV remodeling, blood biomarkers, and histological parameters, and functional capacity than each parameter alone. A combined, multi-parametric approach with ECV and LGE allows efficient stratification of AS patients according to the response of the myocardial collagen matrix [51].

CMR techniques may be more sensitive than the conventional measures (LVEF or LV dimensions) to detect structural and functional changes in patients with severe left-sided valvular heart disease. The presence of MF has been associated with little improvement in clinical symptoms and recovery of LV systolic function [50]. On the contrary, Everett et al. [47] suggested that cellular hypertrophy and diffuse fibrosis (mid- wall LGE) progress in a rapid and balanced manner but are reversible after AVR in patients with AS. When mid-wall LGE is first identified, prompt AVR may improve clinical outcomes. According to a prospective trial by Everett et al. [47], in 61 asymptomatic patients (43\% mild, 34\% moderate, and $23 \%$ severe AS), significant increases in peak aortic jet velocity, LVMI, indexed ECV, and LGE mass were observed after 2.1 \pm 0.7 years, with the most rapid progression observed in patients with severe stenosis. Patients with baseline mid-wall LGE ( $n=16$ [26\%], LGE mass of $2.5 \mathrm{~g}$ [0.8 to 4.8]) showed particularly rapid increases in scar burden (78\% [50\% to $158 \%]$ increase in LGE mass per year). In 38 symptomatic patients (age, $66 \pm 8$ years; $76 \%$ men) who had undergone AVR, there was a $19 \%(11 \%$ to $25 \%)$ reduction in LVMI $(P<0.0001)$ and an $11 \%(4 \%$ to $16 \%)$ reduction in indexed ECV $(P=0.003) 0.9$ \pm 0.3 years after surgery. On the contrary, mid-wall LGE ( $n=10$ [26\%]; mass of $3.3 \mathrm{~g}$ [2.6 to 8.0]) did not change after $\operatorname{AVR}(n=10 ; 3.5 \mathrm{~g}$ [2.1 to 8.0]; $P=0.23$ ), with no evidence of regression up to 2 years.

According to data from the British Society of Cardiovascular Magnetic Resonance Valve Consortium, in patients with severe AS, LGE on CMR was independently associated with mortality; its presence was associated with a 2-fold increase in late mortality [52]. Of the 674 patients with severe AS (75 \pm 14 years, $63 \%$ male; AVA, $0.38 \pm 0.14 \mathrm{~cm}^{2} / \mathrm{m}^{2}$; mean gradient $46 \pm 18 \mathrm{~mm}$ Hg; LVEF, $61.0 \% \pm 16.7 \%)$, scars were present in 51\% (18\% infarct-pattern; 33\% non-infarct) [52]. Management involved surgical (SAVR, $\mathrm{n}=399$ ) or transcatheter procedures (TAVI, $n=275$ ). Scar independently predicted allcause $(26.4 \%$ vs. $12.9 \%, \mathrm{P}<0.001)$ and cardiovascular mortality $(15.0 \%$ vs. $4.8 \%, P<0.001)$, regardless of intervention (TAVI, $\mathrm{P}=0.002$; SAVR, $\mathrm{P}=0.026$ [all-cause mortality]). Every $1 \%$ increase in LV myocardial scar burden was associated with $11 \%$ increase in all-cause mortality $(\mathrm{HR}, 1.11 ; 95 \% \mathrm{Cl}$, 1.05 to $1.17 ; \mathrm{P}<0.001$ ) and $8 \%$ increase in cardiovascular mortality (HR, $1.08 ; 95 \% \mathrm{Cl}, 1.01$ to $1.17 ; \mathrm{P}<0.001$ ).

\section{STRAIN}

LV strain can be measured with CMR [49,53-56]. As a simple and practical method, tissue tracking is a promising method for assessing strain and predicting reverse remodeling in severe AS, especially in patients with suboptimal echocardiographic image quality. TAVI and SAVR procedures are associated with comparable decline in rotational LV mechanics at 6 months, with largely unchanged strain and strain rates [56]. On multivariable Cox analysis, baseline middle LV circumfer- 
ential strain was significantly associated with all-cause mortality (HR, 1.03; 95\% Cl, 1.01 to $1.05 ; \mathrm{P}=0.009$ ), independent of age, LVEF, and Society of Thoracic Surgeons (STS) mortality risk score. Receiver operating curve analysis indicated that a mid-LV circumferential strain $>-18.7 \%$ was associated with significantly reduced survival. No significant change in basal or middle LV circumferential strain or diastolic strain rate was seen after either intervention. However, a significant and comparable decline in LV torsion and twist was observed (SAVR: torsion, $14.08 \pm 8.40$ vs. $7.81 \pm 4.51, P<0.001$; twist, $16.17 \pm 7.01$ vs. $12.45 \pm 4.78, P<0.01$; TAVI: torsion, $14.43 \pm$ 4.66 vs. $11.20 \pm 4.62, P<0.001$, twist, $16.08 \pm 5.36$ vs. 12.36 $\pm 5.21, P<0.001$ ), which likely reflects an improvement towards normal physiology after alleviation of AS [56].

According to Hwang et al. [55], in 63 patients with severe AS and normal LV systolic function (EF $>60 \%$ ), LV mass regression had significantly improved after AVR (baseline 145.9 $\pm 37.0 \mathrm{~g} / \mathrm{m}^{2}$ vs. follow-up $\left.97.7 \pm 22.2 \mathrm{~g} / \mathrm{m}^{2}, \mathrm{P}<0.001\right)$. Statistically significant Pearson's correlations with LVMI regression were observed for longitudinal global strain $(r=-0.461$, $P<0.001)$, radial strain $(r=0.391, P=0.002)$, and circumferential strain $(r=-0.334, P=0.009)$. A simple linear regression analysis showed that all strain parameters could predict the amount of LVMI regression $(P<0.05)$ as well as non-contrast $\mathrm{T} 1$ value (beta $=-0.314, \mathrm{P}<0.001$ ) and ECV (beta $=-2.546$, $P=0.038)$. However, $E C V$ had the lowest predictive power (multiple $r^{2}=0.071$ ). Multiple regression analysis showed that strain could independently predict the amount of LVMI regression and the longitudinal global strain (beta $=-3.335$, $\mathrm{P}<0.001)$.

Using dedicated software, assessment of CT-derived LV strain is feasible. In patients treated with TAVI, CT-derived parameters of global myocardial strain can help improve short-term follow-up. Peak 3D global strain measured with CT in 25 TAVI patients showed increased peak global maximum principal strain after TAVI $(0.59 \pm 0.18)$ from pre-TAVI values $(0.46 \pm 0.19)$ [57]

\section{FOUR-DIMENSIONAL FLOW}

Four-dimensional flow (4D flow) MRI applications for aortic valve disease provide an insight into aortic flow pattern, kinetic energy, wall shear stress (WSS) before and after TAVI, and aortic stiffness [3,45,58-64]. 4D flow MRI recorded significantly higher velocities than $2 \mathrm{D}$ phase-contrast MRI $(2.04 \pm$ $0.71 \mathrm{~m} / \mathrm{sec}$ vs. $1.69 \pm 0.79 \mathrm{~m} / \mathrm{sec}, 17.2 \%$ difference, $\mathrm{P}<0.001$ ) and similar velocities as Doppler echocardiography [65].

According to the study of Van Ooij et al. [66], AS significant- ly alters aortic hemodynamics and WSS independent of aortic valve phenotype. In all, 571 subjects underwent 4D flow MRI in the thoracic aorta (210 cusp fusions for right-left [RL] bicuspid aortic valve [BAV], 60 for right-noncoronary [RN] BAV, 245 tricuspid aortic valve patients with aortic dilatation, and 56 healthy controls). In BAV patients without AS, the different cusp fusion phenotypes resulted in distinct differences in eccentric WSS elevation: RL-BAV patients exhibited an increase in WSS of $9 \%$ to $34 \%(P<0.001)$ at the aortic root and along the entire outer curvature of the ascending aorta (AAo), whereas RN-BAV patients showed 30\% WSS increase $(P<0.001)$ at the distal portion of the AAo. WSS in tricuspid aortic valve patients with aortic dilatation without AS was significantly reduced by $21 \%$ to $33 \%(P<0.01)$ in four of six AAo regions. In all patient groups, mild, moderate, and severe AS resulted in a marked increase in regional WSS $(P<$ 0.001). Moderate-to-severe AS further increased WSS magnitude and variability in the AAo [66].

Rodriguez-Palomares et al. [61] analyzed differences in flow patterns and regional axial and circumferential WSS maps between BAV phenotypes and their correlation with AAo dilatation morphotypes. In all, 101 BAV patients (aortic diameter $\leq 45 \mathrm{~mm}$, no severe valvular disease) and 20 healthy subjects were examined by 4D-flow CMR. BAV phenotype was RL in 78 patients and RN in 23. Both BAV phenotypes presented different outflow jet directions and velocity profiles that matched the location of maximum systolic axial WSS. RL-BAV velocity profiles and maximum axial WSS were homogeneously distributed right-anteriorly; however, RNBAV showed variable profiles with a main proximal-posterior distribution shifting anteriorly at mid-distal AAo. Compared to controls, BAV patients presented similar WSS magnitude at proximal, middle, and distal $\mathrm{AAo}(\mathrm{P}=0.764, \mathrm{P}=0.516$, and $P=0.053$, respectively) but low axial and high circumferential WSS components $(P<0.001$ for both, at all aortic levels). Different BAV phenotypes present different flow patterns with an anterior distribution in RL-BAV, whereas RN-BAV patients present a predominant posterior outflow jet at the sinotubular junction that shifts to anterior or right anterior in the middle and distal AAo. Thus, RL-BAV patients present a high axial WSS at the aortic root while RN-BAV patients present a high circumferential WSS in middle and distal AAo. These results may explain the different AAo dilatation morphotypes in the BAV population [61].

Turbulent kinetic energy (TKE), assessed by 4D flow MRI, is a measure of energy loss in disturbed flow as it occurs in AS. According to Binter et al. [59], elevated TKE levels imply high 
energy losses associated with BAV and dilated AAo geometries. In their study, 51 patients were divided into two groups (severe and mild/moderate AS) according to their echocardiographic mean pressure gradient. TKE values were integrated over the aortic arch to obtain the peak TKE. Integrating over systole yielded total $T \mathrm{TK}_{\text {sys }}$ and by normalizing for stroke volume, normalized TKE $\mathrm{sys}_{\text {s }}$ was obtained. In patients with dilated AAo, both the peak TKE and total TKE $\mathrm{E}_{\text {sys }}$ were significantly elevated $(P<0.01)$, whereas the mean pressure gradient was significantly low $(P<0.05)$. Patients with $B A V$ also showed significantly increased TKE metrics $(P<0.01)$ although no significant difference was found for mean pressure gradient [59].

TAVI alters AAo blood flow and WSS patterns [60]. Compared to the controls, in TAVI patients, abnormally elevated WSS was noted in $30 \% \pm 10 \%$ of the AAo wall. Increased WSS was seen along the posterior mid-AAo and the anterior distal AAo in all TAVI patients. TAVI results in eccentric and displaced flow in the middle and distal AAo, whereas blood flow displacement in SAVR patients occurs only in the distal AAo.

\section{CONCLUSION}

In conclusion, CT plays an important role in pre-TAVI evaluation and exclusion of coronary artery disease in AS patients. MRI can provide information on myocardial perfusion and MF in aortic valve patients. The characterization of blood flow patterns with 4D flow MRI may provide insights on the hemodynamics in aortic valve and the aorta in AS patients.

\section{CONFLICTS OF INTEREST}

No potential conflict of interest relevant to this article was reported.

\section{ORCID}

Yeon Hyeon Choe https://orcid.org/0000-0002-9983-048X

Sung Mok Kim https://orcid.org/0000-0001-5190-2328

Sung-Ji Park https://orcid.org/0000-0002-7075-847X

\section{AUTHOR CONTRIBUTIONS}

Conceptualization: YHC.

Methodology: SMK, SJP.

Original draft preparation: YHC.

Review and editing: SMK, SJP.

\section{REFERENCES}

1. Davin L, Dulgheru R, Bernard A, Marchetta S, Pierard LA, Lancellotti P. Multimodality imaging for the diagnosis and assessment of aortic stenosis severity. Expert Rev Cardiovasc Ther 2016;14:1177-88.

2. Wong S, Spina R, Toemoe S, Dhital K. Is cardiac magnetic resonance imaging as accurate as echocardiography in the assessment of aortic valve stenosis? Interact Cardiovasc Thorac Surg 2016;22:480-6.

3. Bax JJ, Delgado V. Advanced imaging in valvular heart disease. Nat Rev Cardiol 2017;14:209-23.

4. Blanken CPS, Farag ES, Boekholdt SM, Leiner T, Kluin J, Nederveen AJ, et al. Advanced cardiac MRI techniques for evaluation of left-sided valvular heart disease. J Magn Reson Imaging 2018;48:318-29.

5. Kamperidis V, Delgado V, van Mieghem NM, Kappetein AP, Leon MB, Bax JJ. Diagnosis and management of aortic valve stenosis in patients with heart failure. Eur J Heart Fail 2016;18:469-81.

6. Bak SH, Kim SM, Park SJ, Kim MY, Kim HJ, Lee SC, et al. Semiautomated analysis of aortic stenosis parameters on velocity-encoded phase-contrast MR images in patients with severe aortic stenosis: a comparison with echocardiography. Cardiovasc Imaging Asia 2017;1:78-85.

7. Mo YH, Kuo YS, Wang YC, Wu MT. Updates on transcatheter aortic valve replacement and the role of multi-detector computed tomography: what a radiologist should know. Cardiovasc Imaging Asia 2018;2:110-22.

8. ASCI Practice Guideline Working Group, Beck KS, Kim JA, Choe YH, Hian SK, Hoe J, et al. 2017 Multimodality appropriate use criteria for noninvasive cardiac imaging: expert consensus of the Asian Society of Cardiovascular Imaging. Korean J Radiol 2017;18:871-80.

9. Annoni AD, Andreini D, Pontone G, Mancini ME, Formenti A, Mushtaq S, et al. CT angiography prior to TAVI procedure using third-generation scanner with wide volume coverage: feasibility, renal safety and diagnostic accuracy for coronary tree. Br J Radiol 2018;91:20180196.

10. Dankerl P, Hammon M, Seuss H, Trobs M, Schuhbaeck A, Hell MM, et al. Computer-aided evaluation of low-dose and low-contrast agent third-generation dual-source CT angiography prior to transcatheter aortic valve implantation (TAVI). Int J Comput Assist Radiol Surg 2017;12:795802.

11. Hachulla AL, Noble S, Ronot M, Guglielmi G, de Perrot T, Montet X, et al. Low iodine contrast injection for CT ac- 


\section{PRECISION AND FUTURE MIEDICINE}

$\mathrm{CT}$ and MRI of aortic valve stenosis

quisition prior to transcatheter aortic valve replacement: aorta assessment and screening for coronary artery disease. Acad Radiol 2019;26:e150-60.

12. Kim JY, Suh YJ, Chang S, Im DJ, Hong YJ, Lee HJ, et al. Feasibility of a single-beat prospective ECG-gated cardiac CT for comprehensive evaluation of aortic valve disease using a 256-detector row wide-volume CT scanner: an initial experience. Int J Cardiovasc Imaging 2018;34:293300.

13. Mata-Mbemba D, Labani A, El Ghannudi S, Jeung MY, Ohlmann P, Roy C, et al. 320-Row CT transcatheter aortic valve replacement planning with a single reduced contrast media bolus injection. PLoS One 2018;13:e0204145.

14. Matsumoto S, Yamada Y, Hashimoto M, Okamura T, Yamada M, Yashima F, et al. CT imaging before transcatheter aortic valve implantation (TAVI) using variable helical pitch scanning and its diagnostic performance for coronary artery disease. Eur Radiol 2017;27:1963-70.

15. Talei Franzesi CR, Ippolito D, Riva L, Fior D, Cangiotti C, Sironi S. Diagnostic value of iterative reconstruction algorithm in low $\mathrm{kV} \mathrm{CT}$ angiography (CTA) with low contrast medium volume for transcatheter aortic valve implantation (TAVI) planning: image quality and radiation dose exposure. Br J Radiol 2018;91:20170802.

16. Kok M, Turek J, Mihl C, Reinartz SD, Gohmann RF, Nijssen $\mathrm{EC}$, et al. Low contrast media volume in pre-TAVI CT examinations. Eur Radiol 2016;26:2426-35.

17. Felmly LM, De Cecco CN, Schoepf UJ, Varga-Szemes A, Mangold S, McQuiston AD, et al. Low contrast medium-volume third-generation dual-source computed tomography angiography for transcatheter aortic valve replacement planning. Eur Radiol 2017;27:1944-53.

18. Kang EJ. Clinical applications of wide-detector CT scanners for cardiothoracic imaging: an update. Korean J Radiol 2019;20:1583-96.

19. Maragiannis D, Jackson MS, Flores-Arredondo JH, Autry $\mathrm{K}$, Schutt RC, Alvarez PA, et al. Functional assessment of bioprosthetic aortic valves by CMR. JACC Cardiovasc Imaging 2016;9:785-93.

20. Rogers T, Waksman R. Role of CMR in TAVR. JACC Cardiovasc Imaging 2016;9:593-602.

21. Faletti R, Gatti M, Salizzoni S, Bergamasco L, Bonamini R, Garabello D, et al. Cardiovascular magnetic resonance as a reliable alternative to cardiovascular computed tomography and transesophageal echocardiography for aortic annulus valve sizing. Int J Cardiovasc Imaging 2016;32:125563.
22. Yudi MB, Sharma SK, Tang GHL, Kini A. Coronary angiography and percutaneous coronary intervention after transcatheter aortic valve replacement. J Am Coll Cardiol 2018;71:1360-78.

23. Chaikriangkrai K, Jhun HY, Shantha GP, Abdulhak AB, Tandon R, Alqasrawi M, et al. Diagnostic accuracy of coronary computed tomography before aortic valve replacement: systematic review and meta-analysis. J Thorac Imaging 2018;33:207-16.

24. Rossi A, De Cecco CN, Kennon SR, Zou L, Meinel FG, Toscano $\mathrm{W}$, et al. CT angiography to evaluate coronary artery disease and revascularization requirement before trans-catheter aortic valve replacement. J Cardiovasc Comput Tomogr 2017;11:338-46.

25. Ahn JH, Kim SM, Park SJ, Jeong DS, Woo MA, Jung SH, et al. Coronary microvascular dysfunction as a mechanism of angina in severe AS: prospective adenosine-stress CMR study. J Am Coll Cardiol 2016;67:1412-22.

26. Wang Y, Wang M, Song G, Wang W, Lv B, Wang H, et al. Optimal pre-TAVR annulus sizing in patients with bicuspid aortic valve: area-derived perimeter by $\mathrm{CT}$ is the best-correlated measure with intraoperative sizing. Eur Radiol 2019; 29:259-69.

27. Casset C, Jankowski A, Bertrand B, Saunier C, Piliero N, Rodiere $\mathrm{M}$, et al. Evaluation of imaging strategy to optimize and improve outcome of transcatheter aortic valvular implantation. Am J Cardiol 2017;120:1633-8.

28. Blanke P, Pibarot P, Hahn R, Weissman N, Kodali S, Thourani $\mathrm{V}$, et al. Computed tomography-based oversizing degrees and incidence of paravalvular regurgitation of a new generation transcatheter heart valve. JACC Cardiovasc Interv 2017;10:810-20.

29. Yang DH, Park SH, Lee K, Kim T, Kim JB, Yun TJ, et al. Applications of three-dimensional printing in cardiovascular surgery: a case-based review. Cardiovasc Imaging Asia 2018;2:166-75.

30. Ripley B, Kelil T, Cheezum MK, Goncalves A, Di Carli MF, Rybicki FJ, et al. 3D printing based on cardiac CT assists anatomic visualization prior to transcatheter aortic valve replacement. J Cardiovasc Comput Tomogr 2016;10:28-36.

31. Chaturvedi A, Hobbs SK, Ling FS, Chaturvedi A, Knight P. MRI evaluation prior to transcatheter aortic valve implantation (TAVI): when to acquire and how to interpret. Insights Imaging 2016;7:245-54.

32. Mayr A, Klug G, Reinstadler SJ, Feistritzer HJ, Reindl M, Kremser $C$, et al. Is MRI equivalent to $C T$ in the guidance of TAVR?: a pilot study. Eur Radiol 2018;28:4625-34. 
33. Salaun E, Jacquier A, Theron A, Giorgi R, Lambert M, Jaussaud N, et al. Value of CMR in quantification of paravalvular aortic regurgitation after TAVI. Eur Heart J Cardiovasc Imaging 2016;17:41-50.

34. Cannao PM, Muscogiuri G, Schoepf UJ, De Cecco CN, Suranyi P, Lesslie VW, et al. Technical feasibility of a combined noncontrast magnetic resonance protocol for preoperative transcatheter aortic valve replacement evaluation. J Thorac Imaging 2018;33:60-7.

35. Papanastasiou CA, Kokkinidis DG, Jonnalagadda AK, Oikonomou EK, Kampaktsis PN, Garcia MJ, et al. Meta-analysis of transthoracic echocardiography versus cardiac magnetic resonance for the assessment of aortic regurgitation after transcatheter aortic valve implantation. Am J Cardiol 2019;124:1246-51.

36. Tribouilloy C, Bohbot Y, Marechaux S, Debry N, Delpierre $\mathrm{Q}$, Peltier M, et al. Outcome implication of aortic valve area normalized to body size in asymptomatic aortic stenosis. Circ Cardiovasc Imaging 2016;9:e005121.

37. Nies R, Pfister R, Kuhr K, Michels G. Inconsistency in hemodynamic characterization of severe aortic stenosis. Int J Cardiol 2015;197:309-11.

38. Annabi MS, Clisson M, Clavel MA, Pibarot P. Workup and management of patients with paradoxical low-flow, low-gradient aortic stenosis. Curr Treat Options Cardiovasc Med 2018;20:49.

39. Shah RG, Novaro GM, Blandon RJ, Whiteman MS, Asher $\mathrm{CR}$, Kirsch J. Aortic valve area: meta-analysis of diagnostic performance of multi-detector computed tomography for aortic valve area measurements as compared to transthoracic echocardiography. Int J Cardiovasc Imaging 2009;25:601-9.

40. Clavel MA, Malouf J, Messika-Zeitoun D, Araoz PA, Michelena HI, Enriquez-Sarano M. Aortic valve area calculation in aortic stenosis by $\mathrm{CT}$ and Doppler echocardiography. JACC Cardiovasc Imaging 2015;8:248-57.

41. Jander N, Wienecke S, Dorfs S, Ruile P, Neumann FJ, Pache $G$, et al. Anatomic estimation of aortic stenosis severity vs "fusion" of data from computed tomography and Doppler echocardiography. Echocardiography 2018;35:777-84.

42. Pawade T, Clavel MA, Tribouilloy C, Dreyfus J, Mathieu T, Tastet $\mathrm{L}$, et al. Computed tomography aortic valve calcium scoring in patients with aortic stenosis. Circ Cardiovasc Imaging 2018;11:e007146.

43. Mantini C, Di Giammarco G, Pizzicannella J, Gallina S, Ricci F, D'Ugo E, et al. Grading of aortic stenosis severity: a head-to-head comparison between cardiac magnetic res- onance imaging and echocardiography. Radiol Med 2018; 123:643-54.

44. Da Silveira JS, Smyke M, Rich AV, Liu Y, Jin N, Scandling D, et al. Quantification of aortic stenosis diagnostic parameters: comparison of fast 3 direction and 1 direction phase contrast CMR and transthoracic echocardiography. J Cardiovasc Magn Reson 2017;19:35.

45. Donati F, Myerson S, Bissell MM, Smith NP, Neubauer S, Monaghan MJ, et al. Beyond Bernoulli: improving the accuracy and precision of noninvasive estimation of peak pressure drops. Circ Cardiovasc Imaging 2017;10:e005207.

46. Bing R, Cavalcante JL, Everett RJ, Clavel MA, Newby DE, Dweck MR. Imaging and impact of myocardial fibrosis in aortic stenosis. JACC Cardiovasc Imaging 2019;12:283-96.

47. Everett RJ, Tastet L, Clavel MA, Chin CW, Capoulade R, Vassiliou VS, et al. Progression of hypertrophy and myocardial fibrosis in aortic stenosis: a multicenter cardiac magnetic resonance study. Circ Cardiovasc Imaging 2018;11:e007451.

48. Geiger J, Rahsepar AA, Suwa K, Powell A, Ghasemiesfe A, Barker AJ, et al. 4D flow MRI, cardiac function, and T1-mapping: association of valve-mediated changes in aortic hemodynamics with left ventricular remodeling. J Magn Reson Imaging 2018;48:121-31.

49. Mathew RC, Loffler Al, Salerno M. Role of cardiac magnetic resonance imaging in valvular heart disease: diagnosis, assessment, and management. Curr Cardiol Rep 2018;20:119.

50. Podlesnikar T, Delgado V, Bax JJ. Cardiovascular magnetic resonance imaging to assess myocardial fibrosis in valvular heart disease. Int J Cardiovasc Imaging 2018;34:97-112.

51. Treibel TA, Lopez B, Gonzalez A, Menacho K, Schofield RS, Ravassa S, et al. Reappraising myocardial fibrosis in severe aortic stenosis: an invasive and non-invasive study in 133 patients. Eur Heart J 2018;39:699-709.

52. Musa TA, Treibel TA, Vassiliou VS, Captur G, Singh A, Chin C, et al. Myocardial scar and mortality in severe aortic stenosis. Circulation 2018;138:1935-47.

53. Buckert D, Tibi R, Cieslik M, Radermacher M, Qu YY, Rasche $\mathrm{V}$, et al. Myocardial strain characteristics and outcomes after transcatheter aortic valve replacement. Cardiol J 2018;25:203-12.

54. Burris NS, Lima APS, Hope MD, Ordovas KG. Feature tracking cardiac MRI reveals abnormalities in ventricular function in patients with bicuspid aortic valve and preserved ejection fraction. Tomography 2018;4:26-32.

55. Hwang JW, Kim SM, Park SJ, Cho EJ, Kim EK, Chang SA, et al. Assessment of reverse remodeling predicted by myo- 
cardial deformation on tissue tracking in patients with severe aortic stenosis: a cardiovascular magnetic resonance imaging study. J Cardiovasc Magn Reson 2017;19:80.

56. Musa TA, Uddin A, Swoboda PP, Fairbairn TA, Dobson LE, Singh A, et al. Cardiovascular magnetic resonance evaluation of symptomatic severe aortic stenosis: association of circumferential myocardial strain and mortality. J Cardiovasc Magn Reson 2017;19:13.

57. Marwan M, Ammon F, Bittner D, Rother J, Mekkhala N, Hell $M$, et al. CT-derived left ventricular global strain in aortic valve stenosis patients: a comparative analysis pre and post transcatheter aortic valve implantation. J Cardiovasc Comput Tomogr 2018;12:240-4.

58. Bannas P, Lenz A, Petersen J, Sinn M, Adam G, Reichenspurner $\mathrm{H}$, et al. Normalization of transvalvular flow patterns after bicuspid aortic valve repair: insights from four-dimensional flow cardiovascular magnetic resonance imaging. Ann Thorac Surg 2018;106:e319-20.

59. Binter C, Gotschy A, Sundermann SH, Frank M, Tanner FC, Luscher TF, et al. Turbulent kinetic energy assessed by multipoint 4-dimensional flow magnetic resonance imaging provides additional information relative to echocardiography for the determination of aortic stenosis severity. Circ Cardiovasc Imaging 2017;10:e005486.

60. Farag ES, Vendrik J, van Ooij P, Poortvliet QL, van Kesteren F, Wollersheim LW, et al. Transcatheter aortic valve replacement alters ascending aortic blood flow and wall shear stress patterns: a 4D flow MRI comparison with agematched, elderly controls. Eur Radiol 2019;29:1444-51.

61. Rodriguez-Palomares JF, Dux-Santoy L, Guala A, Kale R, Maldonado G, Teixido-Tura G, et al. Aortic flow patterns and wall shear stress maps by 4D-flow cardiovascular magnetic resonance in the assessment of aortic dilatation in bicuspid aortic valve disease. J Cardiovasc Magn Reson 2018;20:28.

62. Ha H, Kim GB, Kweon J, Huh HK, Lee SJ, Koo HJ, et al. Turbulent kinetic energy measurement using phase contrast MRI for estimating the post-stenotic pressure drop: in vitro validation and clinical application. PLoS One 2016;11:e0151540.

63. Ha H, Kim GB, Kweon J, Lee SJ, Kim YH, Lee DH, et al. Hemodynamic measurement using four-dimensional phase-contrast MRI: quantification of hemodynamic parameters and clinical applications. Korean J Radiol 2016;17:445-62.

64. Garcia J, Barker AJ, Markl M. The role of imaging of flow patterns by 4D flow MRI in aortic stenosis. JACC Cardiovasc Imaging 2019;12:252-66.

65. Rose MJ, Jarvis K, Chowdhary V, Barker AJ, Allen BD, Robinson JD, et al. Efficient method for volumetric assessment of peak blood flow velocity using 4D flow MRI. J Magn Reson Imaging 2016;44:1673-82.

66. Van Ooij P, Markl M, Collins JD, Carr JC, Rigsby C, Bonow $\mathrm{RO}$, et al. Aortic valve stenosis alters expression of regional aortic wall shear stress: new insights from a 4-dimensional flow magnetic resonance imaging study of 571 subjects. J Am Heart Assoc 2017;6:e005959. 